\title{
Research on Novel Remote Field Eddy Current Testing System for Axial Crack Detection with High Resolution
}

\author{
XU Xiao-Jie \\ Institute of Information and Navigation, Air Force Engineering University Xi'an, China, 710077
}

\begin{abstract}
Remote field eddy current was an effective non-destructive testing technology for tubular structure such as pipes and tubes. Firstly, experiment results using conventional sensor, the reason of poor sensitivity was analyzed and discussed by finite element simulation. Secondly, novel high resolution sensor with orthogonal magnetic field and self-differential mode pick-up coil was proposed. At last, proposed senor was verified experimentally using various type defects, and conclusions were drawn as followed: detection ability and sensitivity of remote field eddy current testing system using proposed senor improved significantly compared with using conventional sensor, especially for axial crack which is not easily to detect using conventional sensor, and sensitivity to various types defects remained almost the same.
\end{abstract}

KEYWORD: Remote Field Eddy Current; Sensors; Orthogonal Magnetic Field; Finite Element Simulation; Detection Sensitivity

\section{INTRODUCTION}

Remote field eddy current (RFEC) technique recently draws more and more attention in nondestructive testing of tubes and pipes for its promising advantages [1]. Fig1 shows conventional schematic diagram of RFEC, which is dependent on two different coupled paths when electromagnetic wave propagate in the pipe.

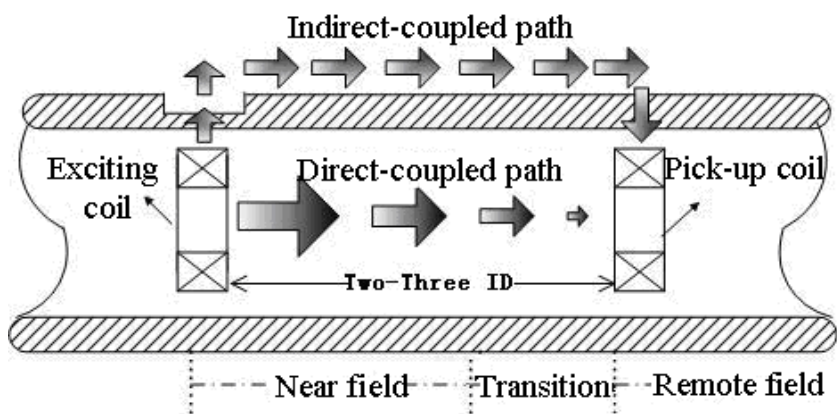

Fig1. Schematic diagram of remote field eddy current

However, further applications of remote field eddy current testing were restricted by disadvantages such as weak signal, poor sensitivity to axial crack. In this paper, we investigate novel sensor design to overcome these disadvantages [2].

\section{SENSITIVITY ANALYSIS}

\subsection{Axial crack detection ability of conventional RFEC sensor}

Fig2 shows conventional remote field eddy current sensor usually adopts a coaxial solenoid coil excited by sinusoid to generate exciting field, and adopts another solenoid coil placed at about 2 3times pipes inner diameter to pick up field[3].

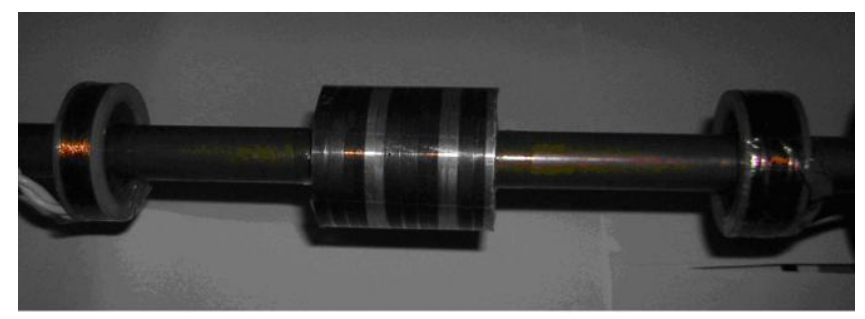

Fig2. Conventional sensor prototype of RFEC testing

To verify detection ability and sensitivity of conventional sensor, two classes defects are manufactured in a section of ferromagnetic tube with $70 \mathrm{~mm}$ inner diameter, $82 \mathrm{~mm}$ outer diameter and $6 \mathrm{~mm}$ wall thickness. First class defects shown in Fig 5 include: circumferential and axial crack with $10 \mathrm{~mm}$ length and $2 \mathrm{~mm}$ width, circular defect with $6 \mathrm{~mm}$ diameter; second class defect shown in Fig.4 include: circumferential and axial crack with $10 \mathrm{mmlength}$ and $0.5 \mathrm{~mm}$ width, circular defect with $3 \mathrm{~mm}$ diameter. All defects are manufactured with depth of $3 \mathrm{~mm}$, equal to $50 \%$ tube wall thickness. 

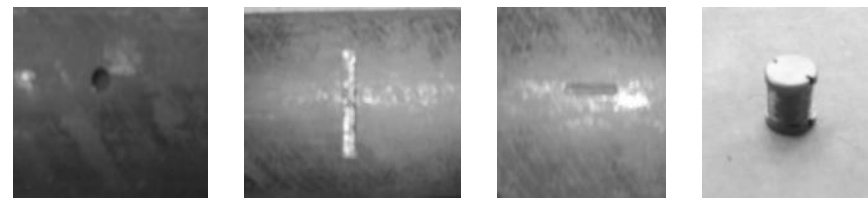

(a) Circular (b) circumferential

(c) axial (d) pick-up coil

Fig3. Photography of first class defects and pick-up coil

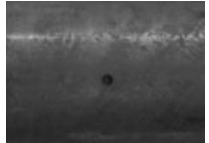

(a) Circular

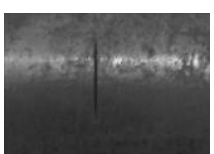

(b) circumferential

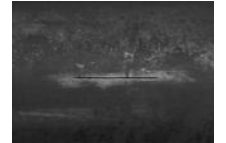

(c) axial
Fig4. Photography of second class defects

Fig 5 shows the experiment results of first class defects. All of defects can be detected and identified clearly, although sensitivity to axial crack is relative lower than circumferential crack and circular defect.

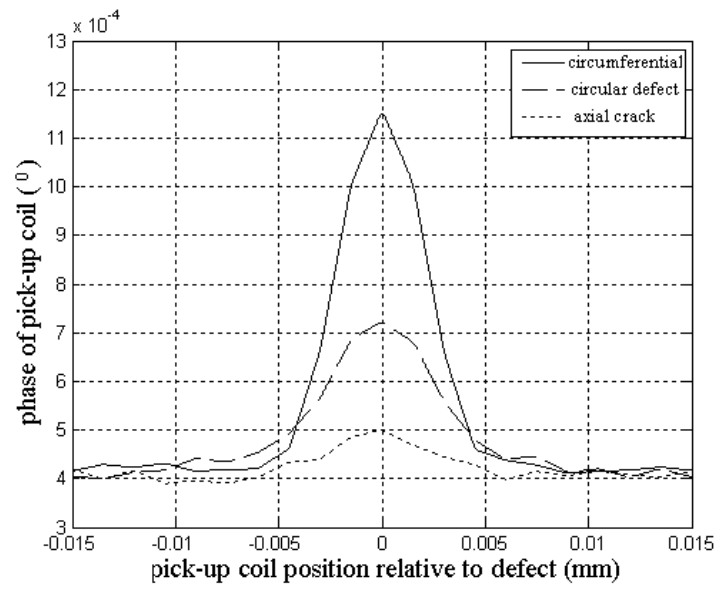

Fig 5. Experiment results for first class defects

However, with reduction of size of defect, all of defects detection ability decreases, especially for axial crack. Fig 6 shows experiment results of second class defects. Amplitude of axial cracks reduce too low to detect it.

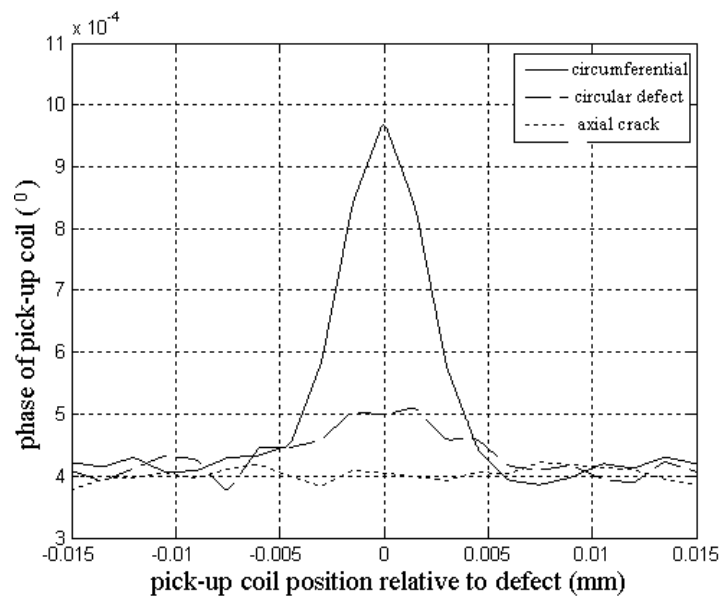

Fig 6. Experiment results for second class defects

\subsection{Finite element simulation and analysis}

Finite element method is proved to be an effective analysis method for eddy current NDT [4] [5] [6], and is adopted to analyze the internal mechanism of poor sensitivity to axial crack rather than defects in other direction.

When excitation frequency is low, the displacement current can be neglected, and the basis governing Maxwell equation will be reduced to following equations:

$$
\begin{aligned}
& \nabla \times \overrightarrow{\boldsymbol{H}}=\overrightarrow{\boldsymbol{J}}+\overrightarrow{\boldsymbol{J}} \boldsymbol{s} \\
& \nabla \times \vec{E}=-\frac{\partial \vec{B}}{\partial t} \\
& \nabla \cdot \overrightarrow{\boldsymbol{B}}=\boldsymbol{0}
\end{aligned}
$$

Where $\vec{J} s$ is source current density, $\vec{J}$ is eddy current density, $\vec{B}$ and $\vec{H}$ are magnetic flux density and magnetic field intensity, $\vec{E}$ is electric field intensity.

Introducing magnetic potential vector $\vec{A}$, which is governed by $\vec{B}=\nabla \times \vec{A}$, the final governing equation is derived from equation (1) (3):

$$
\nabla \times \frac{1}{\mu} \nabla \times \vec{A}=-\sigma \frac{\partial \vec{A}}{\partial t}+\vec{J} s
$$

In harmonic excitation, whose angular frequency is $\omega$, and considering coulomb gauge $\nabla \cdot \vec{A}=0$, Equation(5) is rewritten as:

$$
\left(\frac{1}{\mu}\right) \nabla^{2} \vec{A}=-\vec{J} S+j \omega \sigma \vec{A}
$$

Then we obtain magnetic potential vector $\vec{A}$ by using FEM to mesh and work out Equation (5), therefore magnetic flux density $\vec{B}$ and other wanted quantity can be deduced from it.

Fig 7 shows axial component of magnetic flux density around circumferential crack, and Fig 8 shows axial component of magnetic flux density around axial crack. Distribution caused by circumferential cracks is five times high than axial. It is perhaps because conventional RFEC sensor using coaxial solenoid coil, which mainly generate axial magnetic and induct axial eddy current which is more sensitive to circumferential cracks

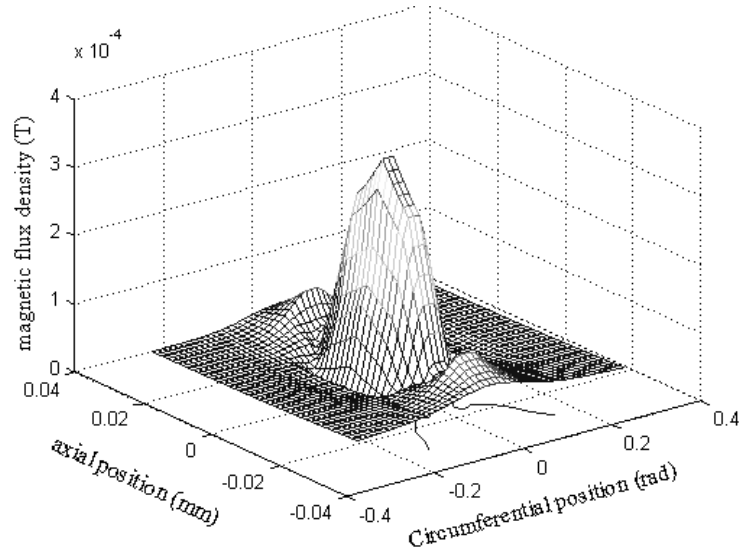

Fig 7. Axial component of magnetic flux density around circumferential crack 


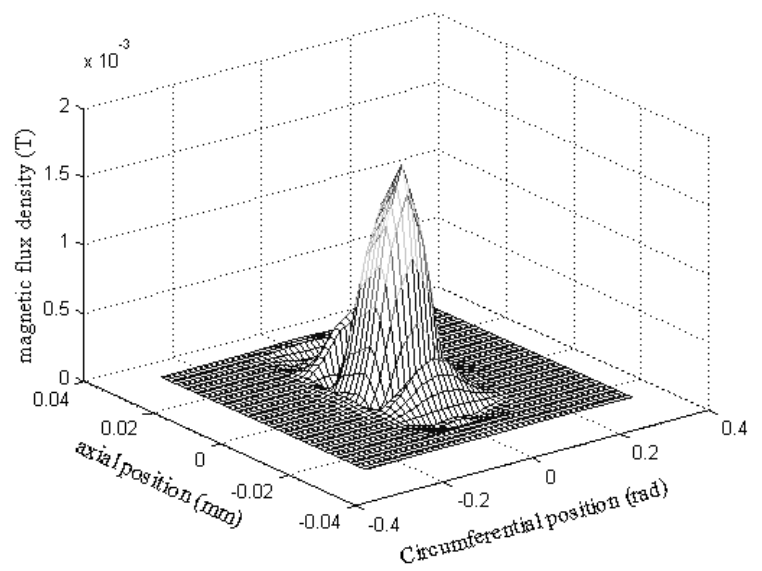

Fig 8. Axial component of magnetic flux density around axial crack

To further verify it, Fig 9 and Fig 10shows circumferential component of magnetic flux density around axial and circumferential crack. Contrary to the previous case, Distribution caused by axial crack is four times high than circumferential crack.

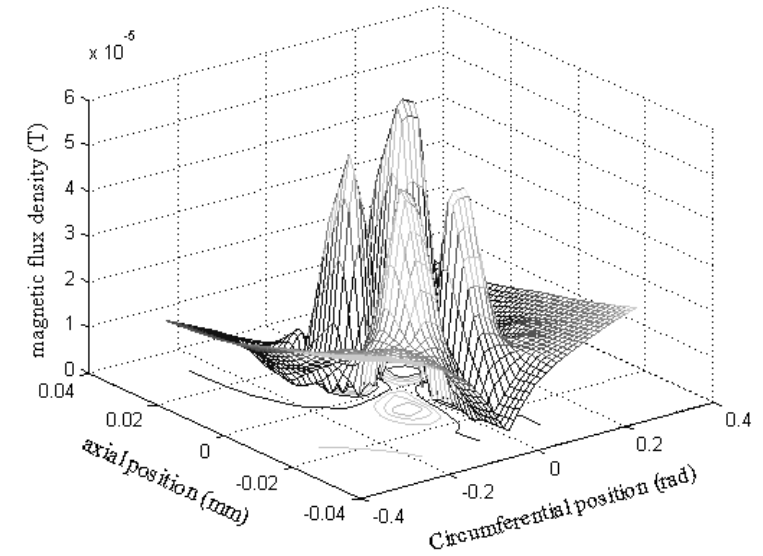

Fig9. Circumferential component of magnetic flux density around circumferential crack

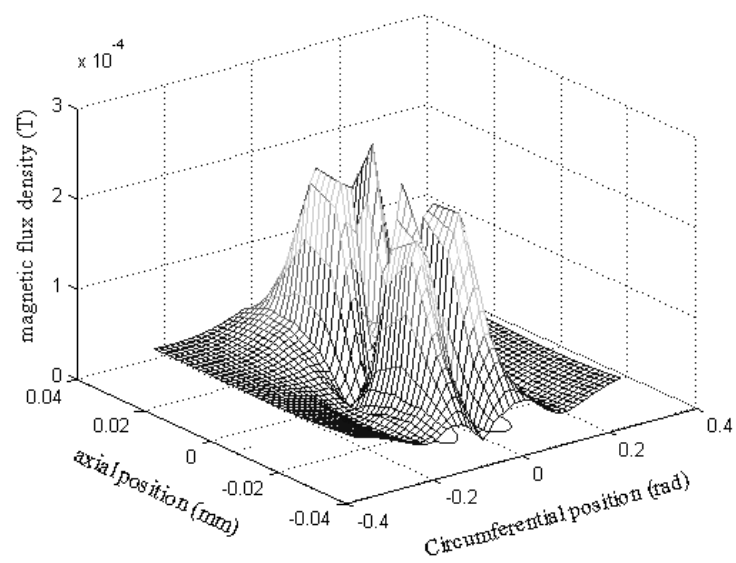

Fig10. Circumferential component of magnetic flux density around axial crack

From above analysis, conclusion can be drawn as followed: The most appropriate sensor configuration should be orthogonal magnetic field combined with axial and circumferential exciting, and specified pick-up coil should be adopted to detect it.

\section{NOVEL SENSOR AND EXPERIMENT SYSEM DESING}

Fig 11 shows novel sensor with orthogonal magnetic field. The sensor consists of exciting coil using to generate orthogonal magnetic field, centering device using to reduce influence of sensor jitter or offcenter, different type pick-up coils with adjustable base to detect field disturbance caused by defect, screw using to adjust the distance between the exciting coil and pick-up coil, and shielding facility using to reduce length of sensor.

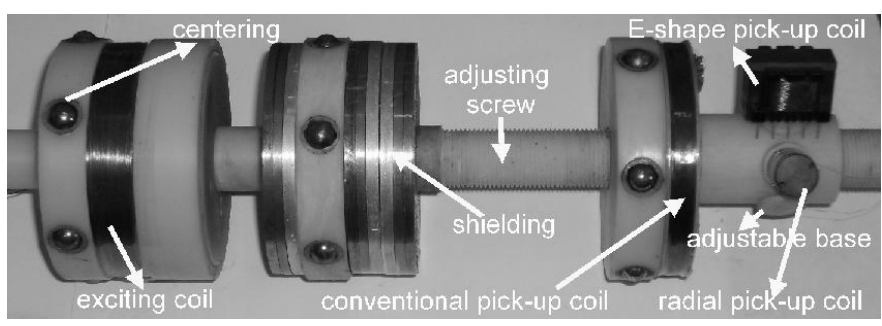

Fig 11 photography of new high resolution sensor

Fig 12 shows orthogonal magnetic field exciting coils and specified pick-up coil. Novel sensor adds a coaxial cylindrical conductor inner the conventional solenoid coil. When AC current was applied between its two ends, due to skin effect, it will be collected on its surface [7], and then spatial circumferential magnetic field will be generated, which will combine with the conventional axial magnetic field to generate orthogonal magnetic field. A new type differential mode pick-up coil with Eshape magnetic core is adopted, which is proposed firstly to detect cracks in aircraft multilayer structure, and is named as "self-differential" [8] [9].
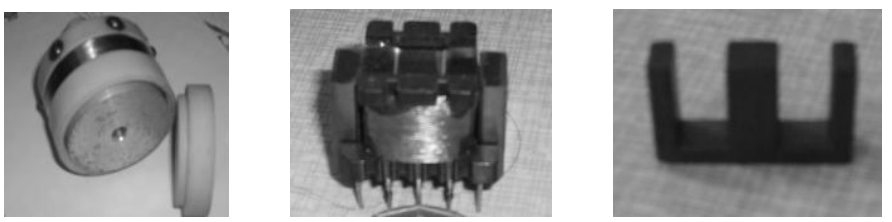

Fig 12 photography of exciting and pick-up coil

Fig 13 shows the experiment system to test and verify the proposed sensor.

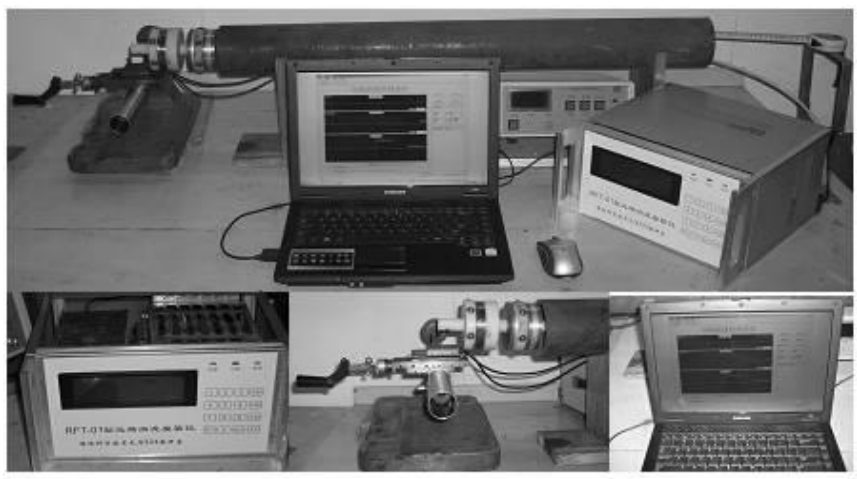

Fig 13 photography of experiment installation 


\section{EXPERIMENT RESULTS}

Test specimen is same to above mentioned, and three types of defects are manufactured with different depth of $15 \%, 20 \%, 40 \%, 60 \%$ tube wall thickness, including circumferential and axial crack with $10 \mathrm{~mm}$ length and $0.5 \mathrm{~mm}$ width, circular defect with $3 \mathrm{~mm}$ diameter.

Fig 14 Fig 16 show experiment results. All of defects can be detected; especially, the axial crack with depth of $20 \%$ wall thickness, which is very difficult to detect using conventional sensor, can be detected and identified clearly. At the same time, the proposed sensor has almost same sensitivity to cracks in different direction: $6.7^{0}$ phase difference for axial crack, $6.2^{0}$ phase difference for circumferential crack.

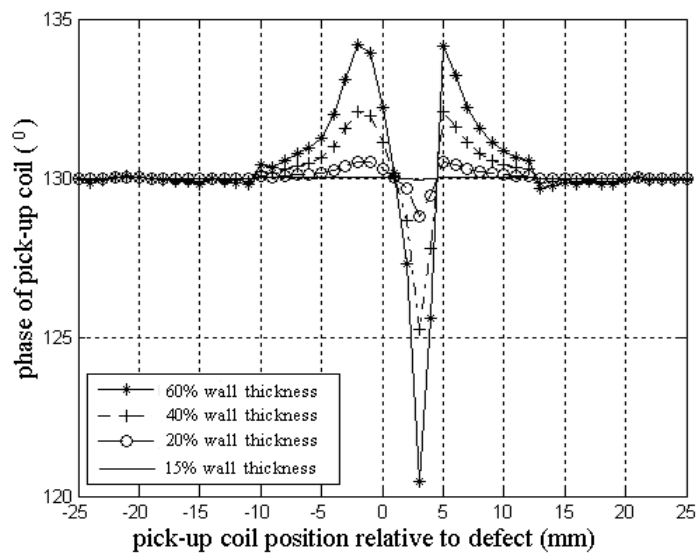

Fig 14 experiment result of axial cracks

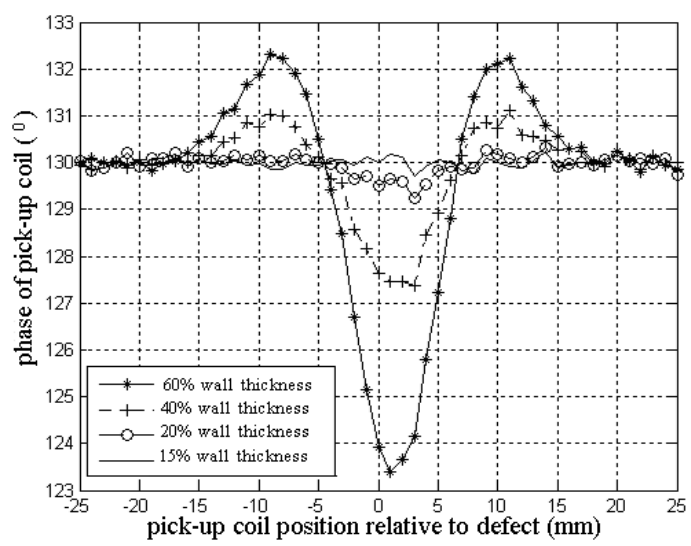

Fig 15 experiment result of axial cracks

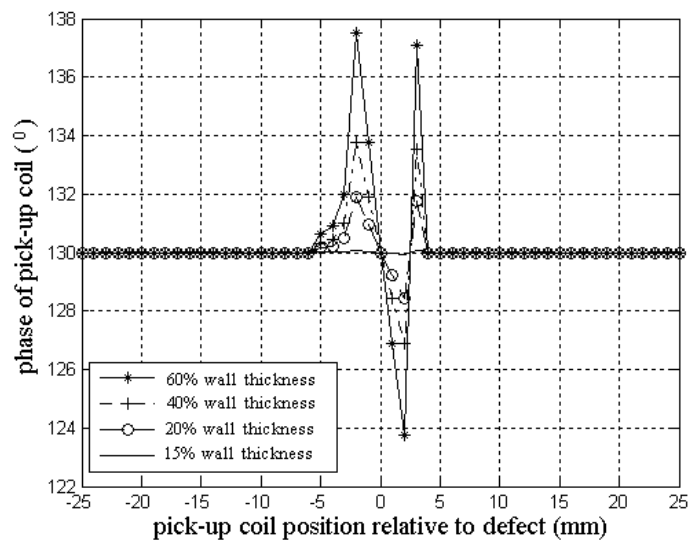

Fig 16 experiment result of circumferential cracks

\section{CONCLUSIONS}

A novel sensor based on orthogonal magnetic field for remote eddy current testing is proposed. After analysis of detection ability and sensitivity of conventional sensor, finite element method is used to simulate and declare the inner mechanism for poor sensitivity to axial crack. By adding a coaxial cylindrical conductor inner conventional solenoid coil, which is considered as the most appropriate configuration to generate orthogonal magnetic field for tubular structures, proposed sensor and experiment system is designed and realized. Experiment results show that proposed sensor has high resolution especially to axial crack which is difficult to detect by conventional sensor, and similar sensitivity to various type of detects.

\section{ACKNOWLEDGEMENT}

Thanks grateful to support by The Chinese National Natural Science Fund (51107148) and The Natural Science Foundation of Shaanxi Province (2011JQ7006)

\section{REFERENCES}

[1] Noriyasu Kobayashi, Souichi Ueno Satoshi Nagai, Makoto Ochiai, Noboru Jimbo.2011.Remote field eddy current testing for steam generator inspection of fast reactor, Nuclear Engineering and Design 241(11):4643-4648.

[2] Atherton D L.1995. Remote Field Eddy Current inspection. IEEE Transactions on Magnetic 31(8):4643-4648.

[3] Young-Joo Kim, Seung-Seok Lee.2012. Eddy current probes of inclined coils for increased detectability of circumferential cracks in tubing, NDT \& E International 49 (1): $77-82$

[4] Junjun Xin, Naiguang Lei, Lalita Udpa, Satish S. Udpa.2013. Rotating field eddy current probe with bobbin pickup coil for steam generator tubes inspection, $N D T \& E$ International, 54(1): 45-55.

[5] Zhen Mao Chen, Mihai Rebican, Kenzo Miya.2005.Three dimensional simulation of remote field ECT using the $\mathrm{Ar}$ method and a new formula for signal calculation. Research in Nondestructive Evaluation 16 (1): 35-53.

[6] Nor taka Yuma, Stephaney Perrine, Kazue Mizuno.2007. Numerical modeling of general cracks from the viewpoint of eddy current simulations. NDT\&E International 40 (7): 577-583.

[7] Xuefei Mao, Yinzhao Lei.2013. Analysis of eddy current interaction between a parallel coil and a ferromagnetic pipe with remanence, NDT \& E International 60(2): 121-126.

[8] Luis S. Rosado, Telmo G. Santos, Pedro M. Ramos, Pedro Vilaça, Moisés Piedade.2012. A differential planar eddy currents probe: Fundamentals, modeling and experimental evaluation, NDT \& E International 51(1): 85-93.

[9] Yushi Sun, Dennis Roach, Harry Zhu.2005. New Advances in Detecting Cracks in Raised-Head Fastener Holes Using Rotational Remote Field Eddy Current Technique, ASNT Fall Conference:17-21. 